Research Article

\title{
New Results on the Geometric-Arithmetic Index
}

\author{
Akbar Jahanbani $\mathbb{D}^{1},{ }^{1}$ Maryam Atapour $\mathbb{D}^{2},{ }^{2}$ and Zhibin Du $\mathbb{D}^{3}$ \\ ${ }^{1}$ Department of Mathematics, Azarbaijan Shahid Madani University, Tabriz, Iran \\ ${ }^{2}$ Department of Mathematics and Computer Science, Basic Science Faculty, University of Bonab, P.O. Box 55513-95133, \\ Bonab, Iran \\ ${ }^{3}$ School of Software, South China Normal University, Foshan, Guangdong 528225, China \\ Correspondence should be addressed to Maryam Atapour; m.atapour@ubonab.ac.ir
}

Received 18 June 2021; Accepted 20 July 2021; Published 31 July 2021

Academic Editor: Ali Ahmad

Copyright (C) 2021 Akbar Jahanbani et al. This is an open access article distributed under the Creative Commons Attribution License, which permits unrestricted use, distribution, and reproduction in any medium, provided the original work is properly cited.

Let $G$ be a graph with vertex set $V(G)$ and edge set $E(G)$. Let $d_{u}$ denote the degree of vertex $u \in V(G)$. The geometric-arithmetic index of $G$ is defined as $G A(G)=\sum_{u v \in E(G)}\left(2 \sqrt{d_{u} d_{v}} /\left(d_{u}+d_{v}\right)\right)$. In this paper, we obtain some new lower and upper bounds for the geometric-arithmetic index and improve some known bounds. Moreover, we investigate the relationships between geometricarithmetic index and several other topological indices.

\section{Introduction}

Let $G$ be a simple graph (i.e., graph without loops and multiple edges) with vertex set $V(G)$ and edge set $E(G)$. The integers $n=|V(G)|$ and $m=|E(G)|$ are the order and the size of the graph $G$, respectively. For $u \in V(G)$, we denote by $d_{u}$ the degree of vertex $u$ in $G$. The minimum and maximum degrees of a graph are denoted by $\delta$ and $\Delta$, respectively.

Graph theory has provided chemists with a variety of useful tools, such as topological indices. A topological index $\operatorname{Top}(G)$ of a graph $G$ is a number with the property that, for every graph $H$ isomorphic to $G$, $\operatorname{Top}(H)=\operatorname{Top}(G)$.

Molecular descriptors play a significant role in mathematical chemistry, especially in QSPR/QSAR investigations. Among them, special place is reserved for so-called topological descriptors. A topological index is a numeric quantity from the structural graph of a molecule.

Usage of topological indices in chemistry began in 1947 when Wiener [1] developed the most widely known topological descriptor, namely, the Wiener index, and used it to determine physical properties of types of alkanes known as paraffin (see, for instance, $[2,3]$ ). The interest of topological indices lies in the fact that they synthesize some of the properties of a molecule into a single number. With this in mind, hundreds of topological indices have been introduced and studied. Topological indices based on the vertex degree play a vital role in mathematical chemistry, and some of them are recognized as tools in chemical research.

Authors are studying various topological descriptors, such as Zagreb indices [4-6], general sum-connectivity index [7, 8], hyper-Zagreb index [9], and harmonic index $[10,11]$. Besides the abovementioned ones, there are other topological descriptors based on end vertex degrees of edges of graphs that have found some applications in QSPR/QSAR research $[2,12,13]$.

The geometric-arithmetic index of a graph is defined in [13] as

$$
\mathrm{GA}(G)=\sum_{u v \in E(G)} \frac{2 \sqrt{d_{u} d_{v}}}{d_{u}+d_{v}}
$$

The geometric-arithmetic index has a number of interesting properties, e.g., see [13]. The lower and upper bounds of the geometric-arithmetic index of connected graphs and the characterizations of graphs for which these bounds are best possible can be found in [13-16]. 
The aim of this paper is to investigate new relationships between the geometric-arithmetic index and other topological indices. In particular, we obtain some lower and upper bounds for the geometric-arithmetic index. Moreover, we improve some known bounds.

\section{Preliminaries}

Let us recall some remarkable lemmas which will be used in this paper.

The first one is a very straightforward observation.
Lemma 1 (see [17]). Let $x$ and $y$ be two positive numbers. Then,

$$
\frac{2 x y}{x+y} \leq \sqrt{x y} \leq \frac{((x+y) / 2)+\sqrt{x y}}{2} \leq \frac{x+y}{2} \leq \sqrt{\frac{x^{2}+y^{2}}{2}} .
$$

The following is the well-known inequality of arithmetic and geometric means.

Lemma 2 (inequality of arithmetic and geometric means, see [18]). Let $x_{1}, \ldots, x_{n}$ be positive numbers. Then,

$$
\frac{n}{\left(1 / x_{1}\right)+\left(1 / x_{2}\right)+\cdots+\left(1 / x_{n}\right)} \leq \sqrt[n]{\prod_{i=1}^{n} x_{i} \leq \frac{x_{1}+x_{2}+\cdots+x_{n}}{n} \leq \sqrt{\frac{x_{1}^{2}+x_{2}^{2}+\cdots+x_{n}^{2}}{n}}}
$$

Lemma 3 (see [19]). Let $a=\left(a_{i}\right)_{i=1}^{n}$ and $\left(b_{i}\right)_{i=1}^{n}$ be two sequences of positive numbers. For any $r \geq 0$,

$$
\sum_{i=1}^{n} \frac{a_{i}^{r+1}}{b^{r}} \geq \frac{\left(\sum_{i=1}^{n} a_{i}\right)^{r+1}}{\left(\sum_{i=1}^{n} b_{i}\right)^{r}}
$$

Lemma 4 (see [20]). Let $r \leq a_{i} \leq R$ for $1 \leq i \leq m$ and $r$ and $R$ be some positive constants. Then,

$$
\sum_{i=1}^{m} a_{i} \sum_{i=1}^{m} \frac{1}{a_{i}} \leq m^{2}\left(1+\frac{1}{4}\left(1-\frac{1+(-1)^{m+1}}{2 m^{2}}\left(\sqrt{\frac{R}{r}}-\sqrt{\frac{r}{R}}\right)^{2}\right)\right) .
$$

Lemma 5 (see [21]). If $a_{1}, a_{2}, \ldots, a_{n}$ and $b_{1}, b_{2}, \ldots, b_{n}$ are positive numbers, where $m_{1} \leq a_{i} \leq N_{1}$ and $m_{2} \leq b_{i} \leq N_{2}$ for each $1 \leq i \leq n$, then

$$
\sum_{i=1}^{n} a_{i}^{2} \sum_{i=1}^{n} b_{i}^{2}-\left(\sum_{i=1}^{n} a_{i} b_{i}\right)^{2} \leq \frac{n^{2}}{4}\left(N_{1} N_{2}+m_{1} m_{2}\right)
$$

Lemma 6 (the Pólya-Szegö inequality, see p. 62 in [22]). Let $a=\left(a_{i}\right)_{i=1}^{n}$ and $\left(b_{i}\right)_{i=1}^{n}$ be two sequences of positive numbers, where $0<m_{1} \leq a_{i} \leq M_{1}$ and $0<m_{2} \leq b_{i} \leq M_{2}$, for $i=1,2, \ldots, n$. Then,

$$
\sum_{i=1}^{n} a_{i}^{2} \sum_{i=1}^{n} b_{i}^{2} \leq \frac{1}{4}\left(\sqrt{\frac{M_{1} M_{2}}{m_{1} m_{2}}}+\sqrt{\frac{m_{1} m_{2}}{M_{1} M_{2}}}\right)^{2}\left(\sum_{i=1}^{n} a_{i} b_{i}\right)^{2} .
$$

\section{Upper Bounds for the Geometric- Arithmetic Index}

In this section, we investigate the relationships between geometric-arithmetic index and some topological indices. Moreover, we obtain some upper bounds for the geometricarithmetic index in terms of order, size, maximum degree, minimum degree, domination number, girth, number of cut edges, and number of pendent vertices.
The first and second Zagreb indices are vertex-degreebased graph invariants defined as

$$
\begin{aligned}
& M_{1}(G)=\sum_{u v \in E(G)}\left(d_{u}+d_{v}\right), \\
& M_{2}(G)=\sum_{u v \in E(G)} d_{u} d_{v} .
\end{aligned}
$$

The quantity $M_{1}$ was first considered in 1972 [6], whereas $M_{2}$ in 1975 [5]. The general Randić index is defined as follows [23]:

$$
R_{\alpha}(G)=\sum_{u v \in E(G)}\left(d_{u} d_{v}\right)^{\alpha}
$$

where $\alpha$ is a real number.

We begin with the establishment of an upper bound for the geometric-arithmetic index in terms of the first Zagreb index and the general Randić index.

Theorem 1. Let $G$ be a graph. Then,

$$
\mathrm{GA}(G) \leq \frac{M_{1}(G)+2 R_{1 / 2}(G)}{4} .
$$

Proof. By Lemma 1, we have

$$
\begin{aligned}
\mathrm{GA}(G) & =\sum_{u v \in E(G)} \frac{2 \sqrt{d_{u} d_{v}}}{d_{u}+d_{v}} \\
& \leq \sum_{u v \in E(G)} \frac{2 d_{u} d_{v}}{d_{u}+d_{v}} \\
& \leq \sum_{u v \in E(G)} \frac{\left(\left(d_{u}+d_{v}\right) / 2\right)+\sqrt{d_{u} d_{v}}}{2} \\
& =\sum_{u v \in E(G)} \frac{d_{u}+d_{v}+2 \sqrt{d_{u} d_{v}}}{4} \\
& =\frac{M_{1}(G)+2 R_{1 / 2}(G)}{4},
\end{aligned}
$$

as desired. 
Using Lemma 1 and an argument similar to the proof of Theorem 1, we can obtain the next result.

Corollary 1. Let $G$ be a graph. Then,

$$
\mathrm{GA}(G) \leq R_{1 / 2}(G) \text {. }
$$

From Lemma 1, we get

$$
R_{1 / 2}(G)=\sum_{u v \in E(G)} \sqrt{d_{u} d_{v}} \leq \sum_{u v \in E(G)} \frac{d_{u}+d_{v}}{2}=\frac{M_{1}(G)}{2} .
$$

Again by Lemma 1, we have

$$
\begin{aligned}
\frac{M_{1}(G)+2 R_{1 / 2}(G)}{4} & =\sum_{u v \in E(G)} \frac{d_{u}+d_{v}+2 \sqrt{d_{u} d_{v}}}{4} \\
& =\sum_{u v \in E(G)} \frac{\left(\left(d_{u}+d_{v}\right) / 2\right)+\sqrt{d_{u} d_{v}}}{2} \\
& \leq \sum_{u v \in E(G)} \frac{d_{u}+d_{v}}{2}=\frac{M_{1}(G)}{2}
\end{aligned}
$$

Hence, we can see that the bounds in Theorem 1 and Corollary 1 improve the bound:

$$
\mathrm{GA}(G) \leq \frac{M_{1}(G)}{2},
$$

established in [15].

The proof of the following result can be found in [23].

Lemma 7 (see [23]). Let $G$ be a graph of size $m$. Then,

$$
R_{\alpha}(G) \leq m\left(\frac{\sqrt{8 m+1}-1}{2}\right)^{2 \alpha},
$$

for $0<\alpha \leq 1$.

Using Corollary 1 and Lemma 7, we can drive the next result.

Corollary 2. Let $G$ be a graph of size $m$. Then,

$$
\mathrm{GA}(G) \leq \frac{m(\sqrt{8 m+1}-1)}{2} .
$$

Lemma 8. Let $x$ and $y$ be two positive numbers. Then,

$$
\begin{aligned}
& \frac{2 \sqrt{x y}}{x+y} \leq 1, \\
& \frac{x+y}{\sqrt{x y}} \geq 2 .
\end{aligned}
$$

Now, we obtain an upper bound for the geometricarithmetic index in terms of the first Zagreb index.
Theorem 2. Let $G$ be a graph of order $n \geq 2$, size $m$, and minimum degree $\delta$. Then,

$$
\mathrm{GA}(G) \leq m-n+\frac{M_{1}(G)}{\delta^{2}}
$$

Proof. Notice that

$$
\sum_{u v \in E(G)} \frac{d_{u}+d_{v}}{d_{u} d_{v}}=\sum_{u v \in E(G)}\left(\frac{1}{d_{u}}+\frac{1}{d_{v}}\right)=n .
$$

By Lemma 8, we have

$$
\begin{aligned}
\mathrm{GA}(G)+n & =\sum_{u v \in E(G)}\left(\frac{2 \sqrt{d_{u} d_{v}}}{d_{u}+d_{v}}+\frac{d_{u}+d_{v}}{d_{u} d_{v}}\right) \\
& \leq \sum_{u v \in E(G)}\left(1+\frac{d_{u}+d_{v}}{d_{u} d_{v}}\right) \\
& =\sum_{u v \in E(G)} 1+\sum_{u v \in E(G)} \frac{d_{u}+d_{v}}{d_{u} d_{v}} \\
& \leq m+\frac{M_{1}(G)}{\delta^{2}}
\end{aligned}
$$

and this implies the desired bound.

A dominating set of a graph is a vertex subset whose closed neighborhood includes all vertices of the graph. The domination number of a graph $G$ is the size of a minimum dominating set.

Theorem 3 (see [24]). Let $T$ be a tree of order $n$ with domination number $\gamma$. Then,

$$
M_{1}(T) \leq(n-\gamma)(n-\gamma+1)+4(\gamma-1) .
$$

By Theorems 2 and 3, we have the following result for trees with the given domination number.

Corollary 3. Let $T$ be a tree of order $n \geq 2$ with domination number $\gamma$. Then,

$$
\mathrm{GA}(T) \leq(n-\gamma)(n-\gamma+1)+4(\gamma-1)-1 .
$$

Since for every two real numbers $x, y$, and $x y \leq\left((x+y)^{2} / 4\right)$, we have the next observation.

Lemma 9. Let $x$ and $y$ be two real numbers, where $x+y \neq 0$. Then, $\left(x y /(x+y)^{2}\right) \leq(1 / 4)$.

Next, we establish an upper bound for the geometricarithmetic index in terms of the second Zagreb index.

Theorem 4. Let $G$ be a graph of size $m$ with maximum degree $\Delta$. Then,

$$
\mathrm{GA}(G) \leq \frac{5 m}{4}-\frac{M_{2}(G)}{4 \Delta^{2}}
$$


Proof. By Lemmas 8 and 9, we have

$$
\begin{aligned}
\mathrm{GA}(G)+\frac{M_{2}(G)}{4 \Delta^{2}} & \leq \sum_{u v \in E(G)}\left(\frac{2 \sqrt{d_{u} d_{v}}}{d_{u}+d_{v}}+\frac{d_{u} d_{v}}{\left(d_{u}+d_{v}\right)^{2}}\right) \\
& \leq \sum_{u v \in E(G)}\left(\frac{2 \sqrt{d_{u} d_{v}}}{d_{u}+d_{v}}+\frac{1}{4}\right) \\
& \leq \sum_{u v \in E(G)}\left(1+\frac{1}{4}\right) \\
& =\frac{5 m}{4}
\end{aligned}
$$

and this implies the desired bound.

In [25], it is proved that, for any tree $T$ of order $n$, $M_{2}(T) \geq 4 n-8$. Using this and Theorem 4 , we obtain the next result.

Corollary 4. Let $T$ be a tree of order $n$ with maximum degree $\Delta$. Then,

$$
\mathrm{GA}(T) \leq \frac{5(n-1)}{4}-\frac{n-2}{\Delta^{2}}
$$

Here, we establish an upper bound for the geometricarithmetic index in terms of the hyper-Zagreb index.

The hyper-Zagreb index is defined as follows [9]:

$$
\operatorname{HM}(G)=\sum_{u v \in E(G)}\left(d_{u}+d_{v}\right)^{2}
$$

Theorem 5. Let $G$ be a graph of order $n$, size $m$, and minimum degree $\delta$. Then,

$$
\mathrm{GA}(G) \leq m-n+\frac{\operatorname{HM}(G)}{2 \delta^{2}} .
$$

Proof. By Inequality (21), we have

$$
\begin{aligned}
\mathrm{GA}(G)+n & \leq \sum_{u v \in E(G)} 1+\sum_{u v \in E(G)} \frac{d_{u}+d_{v}}{d_{u} d_{v}} \\
& \leq \sum_{u v \in E(G)} 1+\sum_{u v \in E(G)} \frac{d_{u}+d_{v}}{\left(2 d_{u} d_{v} /\left(d_{u}+d_{v}\right)\right)} \\
& =\sum_{u v \in E(G)} 1+\sum_{u v \in E(G)} \frac{\left(d_{u}+d_{v}\right)^{2}}{2 d_{u} d_{v}} \\
& \leq m+\frac{\operatorname{HM}(G)}{2 \delta^{2}} .
\end{aligned}
$$

It leads to the desired bound.

The next result is proven in [26].
Theorem 6 (see [26]). Let $G$ be a graph with $n$ vertices and $m$ edges. Then,

$$
\mathrm{HM}(G) \leq \frac{m^{3}(n+1)^{6}}{16 n^{2}(n-1)^{2}}
$$

Theorems 5 and 6 lead to the desired result.

Corollary 5. Let $G$ be a graph of order $n$, size $m$, and minimum degree $\delta$. Then,

$$
\mathrm{GA}(G) \leq m-n+\frac{m^{3}(n+1)^{6}}{32 \delta^{2} n^{2}(n-1)^{2}} .
$$

The redefined third Zagreb index is defined as follows [27]:

$$
\operatorname{Re} Z_{3}(G)=\sum_{u n \in E(G)}\left(d_{u} d_{v}\right)\left(d_{u}+d_{v}\right)
$$

Now, we obtain an upper bound for the geometricarithmetic index in terms of the second Zagreb index, the general Randić index, and the redefined third Zagreb index.

Theorem 7. Let $G$ be a graph with maximum degree $\Delta$ and minimum degree $\delta$. Then,

$$
\mathrm{GA}(G) \leq M_{2}(G)+\frac{R_{1 / 2}(G)}{\delta}-\frac{\operatorname{Re} Z_{3}(G)}{2 \Delta} .
$$

Proof. It is easy to obtain

$$
\begin{aligned}
M_{2}(G)-\mathrm{GA}(G) & =\sum_{u v \in E(G)}\left(d_{u} d_{v}-\frac{2 \sqrt{d_{u} d_{v}}}{d_{u}+d_{v}}\right) \\
& =\sum_{u v \in E(G)}\left(\frac{\left(d_{u}+d_{v}\right) d_{u} d_{v}-2 \sqrt{d_{u} d_{v}}}{d_{u}+d_{v}}\right) \\
& =\sum_{u v \in E(G)} \frac{\left(d_{u}+d_{v}\right) d_{u} d_{v}}{d_{u}+d_{v}}-\sum_{u v \in E(G)} \frac{2 \sqrt{d_{u} d_{v}}}{d_{u}+d_{v}} \\
& \geq \frac{\operatorname{Re} Z_{3}(G)}{2 \Delta}-\frac{R_{1 / 2}(G)}{\delta} .
\end{aligned}
$$

The desired bound follows.

Theorem 8. Let $G$ be a graph of order $n$, size $m$, maximum degree $\Delta$, and minimum degree $\delta$. Then,

$$
\mathrm{GA}(G) \leq \frac{2 m^{2}}{n}\left(1+\frac{1}{4}\left(1-\frac{1+(-1)^{m+1}}{2 m^{2}}\left(\frac{\Delta}{\delta}-\frac{\delta}{\Delta}\right)^{2}\right)\right)
$$

Proof. Now, putting $a_{u v}=\left(2 \sqrt{d_{u} d_{v}} / d_{u}+d_{v}\right)$ for each edge $u v \in E(G), R=(\Delta / \delta)$, and $r=(\delta / \Delta)$ in Lemma 4, we have 


$$
\sum_{u v \in E(G)} \frac{2 \sqrt{d_{u} d_{v}}}{d_{u}+d_{v}} \sum_{u v \in E(G)} \frac{d_{u}+d_{v}}{2 \sqrt{d_{u} d_{v}}} \leq m^{2}\left(1+\frac{1}{4}\left(1-\frac{1+(-1)^{m+1}}{2 m^{2}}\left(\frac{\Delta}{\delta}-\frac{\delta}{\Delta}\right)^{2}\right)\right) .
$$

On the contrary, we have

$$
\frac{n}{2}=\sum_{u v \in E(G)} \frac{d_{u}+d_{v}}{2 d_{u} d_{v}} \leq \sum_{u v \in E(G)} \frac{d_{u}+d_{v}}{2 \sqrt{d_{u} d_{v}}}
$$

Finally, we get the bound by using Inequalities (36) and (37).

The sigma index of $G$ is defined in [28] as

$$
\sigma(G)=\sum_{u v \in E(G)}\left(d_{u}-d_{v}\right)^{2}
$$

Here, we obtain an upper bound for the geometricarithmetic index in terms of the first Zagreb index and the sigma index.

Theorem 9. Let $G$ be a nontrivial graph with maximum degree $\Delta$. Then,

$$
\mathrm{GA}(G) \leq \frac{M_{1}(G)}{2}-\frac{\sigma(G)}{4 \Delta}
$$

Proof. For two real numbers $x$ and $y$, we have that

$$
x y=\frac{1}{4}\left((x+y)^{2}-(x-y)^{2}\right) \text {. }
$$

By (40), we obtain

$$
\begin{aligned}
\mathrm{GA}(G) & =\sum_{u v \in E(G)} \frac{2 \sqrt{d_{u} d_{v}}}{d_{u}+d_{v}} \leq \sum_{u v \in E(G)} \frac{2 d_{u} d_{v}}{d_{u}+d_{v}} \\
& =\sum_{u v \in E(G)} \frac{\left(d_{u}+d_{v}\right)^{2}-\left(d_{u}-d_{v}\right)^{2}}{2\left(d_{u}+d_{v}\right)} \\
& =\frac{1}{2} \sum_{u v \in E(G)}\left(d_{u}+d_{v}\right)-\sum_{u v \in E(G)} \frac{\left(d_{u}-d_{v}\right)^{2}}{2\left(d_{u}+d_{v}\right)} \\
& \leq \frac{1}{2} \sum_{u v \in E(G)}\left(d_{u}+d_{v}\right)-\sum_{u v \in E(G)} \frac{\left(d_{u}-d_{v}\right)^{2}}{4 \Delta} \\
& =\frac{M_{1}(G)}{2}-\frac{\sigma(G)}{4 \Delta},
\end{aligned}
$$

and this implies the desired bound.

The general first $F$-index of a graph $G$ is defined in [29] as

$$
F_{1}^{a}(G)=\sum_{u v \in E(G)}\left(d_{u}^{2}+d_{v}^{2}\right)^{a}
$$

where $a$ is a real number. In particular, $F_{1}^{1}(G)=F(G)$.

Since for every two real numbers $x$ and $y,(x-y)^{2} \geq 0$, and we deduce that, for any graph $G$,

$$
\begin{aligned}
& F(G) \geq 2 M_{2}(G) \\
& \sigma(G)=F(G)-2 M_{2}(G)
\end{aligned}
$$

Using these and Theorem 9, we obtain the next result.

Corollary 6. Let $G$ be a nontrivial graph with maximum degree $\Delta$. Then,

$$
\mathrm{GA}(G) \leq \frac{M_{1}(G)}{2}-\frac{F(G)-2 M_{2}(G)}{4 \Delta} .
$$

From $F(G) \geq 2 M_{2}(G)$, we would like to indicate that the above new bound improves the known bound:

$$
\mathrm{GA}(G) \leq \frac{M_{1}(G)}{2},
$$

which was established in [15].

Now, by using the following result, we want to obtain an upper bound for trees.

Theorem 10 (see [30]). Let $T$ be a tree of order $n$ with independence number $\alpha$. Then,

$$
M_{1}(T) \leq \alpha^{2}-3 \alpha+4 n-4
$$

Here, by Theorems 9 and 10, we obtain the next result.

Corollary 7. Let $T$ be a tree of order $n$ with independence number $\alpha$ and maximum degree $\Delta$. Then,

$$
\mathrm{GA}(T) \leq \frac{\alpha^{2}-3 \alpha+4 n-4}{2}-\frac{\sigma(G)}{4 \Delta}
$$

\section{Lower Bounds for the Geometric- Arithmetic Index}

In this section, we first investigate the relationships between the geometric-arithmetic index and some other topological indices, and then, we obtain some lower bounds for the geometricarithmetic index which improve some well-known bounds.

Theorem 11. Let $G$ be a graph of size $m$ with minimum degree $\delta$. Then,

$$
\mathrm{GA}(G) \geq \frac{4 \delta^{2} m^{2}}{\operatorname{HM}(G)}
$$

Proof. By Lemmas 1 and 2, we have 


$$
\begin{aligned}
\frac{m^{2}}{\mathrm{GA}(G)} & =\frac{m^{2}}{\sum_{u v \in E(G)}\left(2 \sqrt{d_{u} d_{v}} /\left(d_{u}+d_{v}\right)\right)} \\
& \leq \sum_{u v \in E(G)} \frac{d_{u}+d_{v}}{2 \sqrt{d_{u} d_{v}}} \\
& \leq \sum_{u v \in E(G)} \frac{d_{u}+d_{v}}{\left(4 d_{u} d_{v} /\left(d_{u}+d_{v}\right)\right)} \\
& =\sum_{u v \in E(G)} \frac{\left(d_{u}+d_{v}\right)^{2}}{4 d_{u} d_{v}} \\
& \leq \frac{1}{4 \delta^{2}} \sum_{u v \in E(G)}\left(d_{u}+d_{v}\right)^{2} \\
& =\frac{\operatorname{HM}(G)}{4 \delta^{2}} .
\end{aligned}
$$

The result follows.

Here, by Theorems 11 and 6 , we have the next result.

Corollary 8. Let $G$ be a graph of order $n$ and size $m$, with minimum degree $\delta$. Then,

$$
\mathrm{GA}(G) \geq \frac{64 n^{2} \delta^{2}(n-1)^{2}}{m(n+1)^{6}} .
$$

Since for any real numbers $x$ and $y$, it holds that $\left((x+y)^{2} / 4\right) \leq\left(\left(x^{2}+y^{2}\right) / 2\right)$; hence, by this fact and Inequality (49), we can obtain the following result.

Corollary 9. Let $G$ be a graph of size $m$ with minimum degree $\delta$. Then,

$$
\mathrm{GA}(G) \geq \frac{2 \delta^{2} m^{2}}{F(G)}
$$

We start with a lower bound for the geometric-arithmetic index in terms of the general $F$-index.

Theorem 12. Let $G$ be a nontrivial graph of size $m$ with minimum degree $\delta$. Then,

$$
\mathrm{GA}(G) \geq \frac{\sqrt{2} \delta m^{2}}{F_{1}^{1 / 2}(G)}
$$

Proof. Set $r=1, a_{u v}=\sqrt[4]{2 d_{u} d_{v}}$, and $b_{u v}=\sqrt{d_{u}^{2}+d_{v}^{2}}$ for each $u v \in E(G)$. By Lemmas 1 and 3, we have

$$
\begin{aligned}
\mathrm{GA}(G) & =\sum_{u v \in E(G)} \frac{2 \sqrt{d_{u} d_{v}}}{d_{u}+d_{v}} \\
& \geq \sum_{u v \in E(G)} \frac{2 \sqrt{d_{u} d_{v}}}{2 \sqrt{\left(d_{u}^{2}+d_{v}^{2} / 2\right)}} \\
& =\sum_{u v \in E(G)} \frac{\sqrt{2 d_{u} d_{v}}}{\sqrt{d_{u}^{2}+d_{v}^{2}}} \\
& =\sum_{u v \in E(G)} \frac{\left(\sqrt[4]{2 d_{u} d_{v}}\right)^{2}}{\sqrt{d_{u}^{2}+d_{v}^{2}}} \\
& \geq \frac{\left(\sum_{u v \in E(G)} \sqrt[4]{2 d_{u} d_{v}}\right)^{2}}{\sum_{u v \in E(G)} \sqrt{d_{u}^{2}+d_{v}^{2}}} \\
& \geq \frac{\sqrt{2} \delta m^{2}}{F_{1}^{1 / 2}(G)} .
\end{aligned}
$$

The proof is completed.

The harmonic index is defined as follows [11]:

$$
H(G)=\sum_{u v \in E(G)} \frac{2}{d_{u}+d_{v}} .
$$

Theorem 13. Let $G$ be a nontrivial graph of order $n$, size $m$, and minimum degree $\delta$. Then,

$$
\mathrm{GA}(G) \geq \delta(H(G)+n)-2 m .
$$

Proof. Notice that

$$
\begin{aligned}
\mathrm{GA}(G)+2 m & =\sum_{u v \in E(G)} \frac{2 \sqrt{d_{u} d_{v}}}{d_{u}+d_{v}}+\sum_{u \in V(G)} d_{u} \\
& \leq \sum_{u v \in E(G)} \frac{2 \sqrt{d_{u} d_{v}}}{d_{u}+d_{v}}+\sum_{u \in V(G)} \delta \\
& =\sum_{u v \in E(G)} \frac{2 \sqrt{d_{u} d_{v}}}{d_{u}+d_{v}}+n \delta \\
& \leq \delta H(G)+n \delta .
\end{aligned}
$$

The result follows.

Applying (56), we obtain the next results.

Corollary 10. Let $G$ be a nontrivial graph of order $n$, size $m$, and minimum degree $\delta$. Then, 


$$
\mathrm{GA}(G) \geq \frac{R_{1 / 2}(G)}{\Delta}+\delta n-2 m
$$

Corollary 11. Let $G$ be a nontrivial graph of order $n$, size $m$, and minimum degree $\delta$. Then,

$$
\mathrm{GA}(G) \geq \frac{\delta m}{\Delta}+\delta n-2 m .
$$

Theorem 14 (see [31]). Let $G$ be a connected graph of order $n \geq 3$. Then,

$$
H(G) \geq \frac{2(n-1)}{n} .
$$

A cut edge of a graph is an edge whose removal increases the number of connected components of the graph.

Lemma 10 (see [32]). Let $G$ be a connected graph of order $n$ and $k^{\prime}$ cut edges. Then,

$$
m \leq \frac{\left(n-k^{\prime}\right)\left(n-k^{\prime}-1\right)}{2}+k^{\prime}
$$

Now, by Theorems 13 and 14, and Lemma 10, we can obtain the next result.

Corollary 12. Let $G$ be a connected graph of order $n, k^{\prime}$ cut edges, and minimum degree $\delta$. Then,

$$
\mathrm{GA}(G) \geq \delta\left(\frac{2(n-1)}{n}+n\right)-2\left(\frac{\left(n-k^{\prime}\right)\left(n-k^{\prime}-1\right)}{2}+k^{\prime}\right) .
$$

Here, we will use the following particular case of Jensen's inequality.

Lemma 11. Let $f(x)$ be a convex function defined in $x>0$. For $x_{1}, x_{2}, \ldots, x_{m}>0$,

$$
f\left(\frac{x_{1}+x_{2}+\cdots+x_{m}}{m}\right) \leq \frac{1}{m}\left(f\left(x_{1}\right)+f\left(x_{2}\right)+\cdots+f\left(x_{m}\right)\right) .
$$
[8]:

The general sum-connectivity index is defined as follows

$$
\chi_{\alpha}(G)=\sum_{u v \in E(G)}\left(d_{u}+d_{v}\right)^{\alpha} .
$$

Now, we obtain a lower bound for the geometric-arithmetic index in terms of the general sum connectivity index.

Theorem 15. Let $G$ be a graph of size $m$ and minimum degree $\delta$. Then,

$$
\mathrm{GA}(G) \geq \frac{4 \delta^{2} \sqrt{m^{3}}}{\sqrt{\chi_{4}(G)}}
$$

Proof. Since $f(x)=\left(1 / x^{2}\right)$ is a convex function for $x>0$, from Lemmas 1 and 11, we have

$$
\begin{aligned}
\left(\frac{m}{\operatorname{GA}(G)}\right)^{2} & =\left(\frac{m}{\sum_{u v \in E(G)}\left(2 \sqrt{d_{u} d_{v}} /\left(d_{u}+d_{v}\right)\right)}\right)^{2} \\
& \leq \frac{1}{m} \sum_{u v \in E(G)}\left(\frac{d_{u}+d_{v}}{2 \sqrt{d_{u} d_{v}}}\right)^{2} \\
& \leq \frac{1}{m} \sum_{u v \in E(G)}\left(\frac{d_{u}+d_{v}}{\left(4 d_{u} d_{v} /\left(d_{u}+d_{v}\right)\right)}\right)^{2} \\
& =\frac{1}{m} \sum_{u v \in E(G)}\left(\frac{\left(d_{u}+d_{v}\right)^{2}}{4 d_{u} d_{v}}\right)^{2} \\
& \leq \frac{1}{16 m \delta^{4}} \sum_{u v \in E(G)}\left(d_{u}+d_{v}\right)^{4} \\
& =\frac{\chi_{4}(G)}{16 m \delta^{4}},
\end{aligned}
$$

as desired.

Now, we obtain an upper bound for the geometricarithmetic index in terms of the sigma index.

Theorem 16. Let $G$ be a simple connected graph of size $m$ with maximum degree $\Delta, p$ pendent vertices, and minimum nonpendent vertex degree $\delta_{1}$. Then,

$$
\mathrm{GA}(G) \geq \frac{2 p \sqrt{\Delta}}{1+\Delta}+\frac{\sqrt{4(m-p)^{2}-\left(m-p / \delta_{1}^{2}\right)\left(\sigma(G)-p\left(\delta_{1}-1\right)^{2}\right)}}{\sqrt{\left(\Delta+\delta_{1} / 2 \sqrt{\Delta \delta_{1}}\right)}+\sqrt{\left(2 \sqrt{\Delta \delta_{1}} / \Delta+\delta_{1}\right)}} .
$$

Proof. We partition all the edges into two parts: pendent edges and nonpendent edges, so

$$
\mathrm{GA}(G)=\sum_{\substack{u v \in E(G) \\ d_{u}=1}} \frac{2 \sqrt{d_{v}}}{1+d_{v}}+\sum_{\substack{u v \in E(G) \\ d_{u}, d_{v} \neq 1}} \frac{2 \sqrt{d_{u} d_{v}}}{d_{u}+d_{v}} .
$$

On one hand, for the pendent edges, it is not hard to check that $\left(2 \sqrt{d_{v}} / 1+d_{v}\right)$ decreases in $2 \leq d_{v} \leq \Delta$; thus,

$$
\sum_{\substack{u v \in E(G) \\ d_{u}=1}} \frac{2 \sqrt{d_{v}}}{1+d_{v}} \geq \frac{2 p \sqrt{\Delta}}{1+\Delta}
$$

Now, we consider the nonpendent edges. It is easy to see that the function $x+(1 / x)$ gets its maximum value when $x$ attains the maximum or minimum value. From $(\Delta / \delta) \geq\left(d_{u} / d_{v}\right) \geq(\delta / \Delta)$ for all $u$ and $v \in V(G)$, we have

$$
\sqrt{\frac{d_{u}}{d_{v}}}+\sqrt{\frac{d_{v}}{d_{u}}} \leq \sqrt{\frac{\Delta}{\delta}}+\sqrt{\frac{\delta}{\Delta}}
$$


which is equivalent to

$$
\frac{2 \sqrt{\Delta \delta_{1}}}{\Delta+\delta_{1}} \leq \frac{2 \sqrt{d_{u} d_{v}}}{d_{u}+d_{v}} \leq 1
$$

Set $a_{u v}=1$ and $b_{u v}=\left(2 \sqrt{d_{u} d_{v}} / d_{u}+d_{v}\right)$ for each edge $u v \in E(G), M_{1}=m_{1}=M_{2}=1$, and $m_{2}=\left(2 \sqrt{\Delta \delta_{1}} / \Delta+\delta_{1}\right)$ in Lemma 6 , and we have

$$
\begin{aligned}
& \sum_{\substack{u v \in E(G) d_{u}, d_{v} \neq 1}} 1_{\substack{u v \in E(G) d_{u} \\
d_{v} \neq 1}}\left(\frac{2 \sqrt{d_{u} d_{v}}}{d_{u}+d_{v}}\right)^{2} \\
& =(m-p) \sum_{\substack{u v \in E(G) d_{u} \\
d_{v} \neq 1}}\left(1-\left(\frac{d_{u}-d_{v}}{d_{u}+d_{v}}\right)^{2}\right) \\
& \leq \frac{1}{4}\left(\sqrt{\frac{1}{\left(2 \sqrt{\Delta \delta_{1}} / \Delta+\delta_{1}\right)}}+\sqrt{\frac{2 \sqrt{\Delta \delta_{1}}}{\Delta+\delta_{1}}}\right)^{2}\left(\sum_{\substack{u v \in E(G) d_{u}, d_{v} \neq 1}} \frac{2 \sqrt{d_{u} d_{v}}}{d_{u}+d_{v}}\right)^{2},
\end{aligned}
$$

which implies that

$$
\begin{aligned}
\sum_{\substack{u v \in E(G) d_{u}, d_{v} \neq 1}} \frac{2 \sqrt{d_{u} d_{v}}}{d_{u}+d_{v}} & \geq \frac{\sqrt{4(m-p) \sum_{u v \in E(G) d_{u}}\left(1-\left(d_{u}-d_{v} / d_{u}+d_{v}\right)^{2}\right)}}{\sqrt{\left(\Delta+\delta_{1} / 2 \sqrt{\Delta \delta_{1}}\right)}+\sqrt{\left(2 \sqrt{\Delta \delta_{1}} / \Delta+\delta_{1}\right)}} \\
& \geq \frac{\sqrt{4(m-p)^{2}-\left(m-p / \delta_{1}^{2}\right) \sum_{\substack{u v \in E(G) d_{u}, d_{v} \neq 1}}\left(d_{u}-d_{v}\right)^{2}}}{\sqrt{\left(\Delta+\delta_{1} / 2 \sqrt{\Delta \delta_{1}}\right)}+\sqrt{\left(2 \sqrt{\Delta \delta_{1}} / \Delta+\delta_{1}\right)}} \\
& =\frac{\left.\sqrt{4(m-p)^{2}-\left(m-p / \delta_{1}^{2}\right)\left(\sigma(G)-\sum_{u v \in E(G) d_{u}}=1\right.}\left(d_{v}-1\right)^{2}\right)}{\sqrt{\left(\Delta+\delta_{1} / 2 \sqrt{\Delta \delta_{1}}\right)+\sqrt{\left(2 \sqrt{\Delta \delta_{1}} / \Delta+\delta_{1}\right)}}} \\
& \geq \frac{\sqrt{4(m-p)^{2}-\left(m-p / \delta_{1}^{2}\right)\left(\sigma(G)-p\left(\delta_{1}-1\right)^{2}\right)}}{\sqrt{\left(\Delta+\delta_{1} / 2 \sqrt{\Delta \delta_{1}}\right)}+\sqrt{\left(2 \sqrt{\Delta \delta_{1}} / \Delta+\delta_{1}\right)}}
\end{aligned}
$$

Finally, the result follows from (67), (68), and (72).

Next, results are immediate consequences of Theorem 16 with the setting $p=0$.

Corollary 13. For a graph $G$ of size $m$ with maximum degree $\Delta$ and minimum degree $\delta \geq 2$,

$$
\mathrm{GA}(G) \geq \frac{\sqrt{4 m^{2}-\left(m / \delta^{2}\right) \sigma(G)}}{\sqrt{(\Delta+\delta / 2 \sqrt{\Delta \delta})}+\sqrt{(2 \sqrt{\Delta \delta} / \Delta+\delta)}}
$$

Now, we obtain a lower bound for the geometricarithmetic index in terms of the second Zagreb index and the general sum connectivity index.

Theorem 17. Let $G$ be a graph of size $m$, maximum degree $\Delta$, and minimum degree $\delta$. Then,

$$
\mathrm{GA}(G) \geq \sqrt{4 M_{2}(G) \chi_{-2}(G)-\frac{m^{2}}{4} \cdot \frac{\Delta^{2}+\delta^{2}}{\Delta \delta}} .
$$


Proof. By Lemma 5 and putting $a_{u v}=2 \sqrt{d_{u} d_{v}}$, $b_{u v}=\left(1 / d_{u}+d_{v}\right), m_{1}=2 \delta, N_{1}=2 \Delta, m_{2}=(1 / 2 \Delta), \quad$ and $N_{2}=(1 / 2 \delta)$, we have

$$
\sum_{i=1}^{n} 4 d_{u} d_{v} \sum_{i=1}^{n} \frac{1}{\left(d_{u}+d_{v}\right)^{2}}-\left(\sum_{i=1}^{n} \frac{2 \sqrt{d_{u} d_{v}}}{d_{u}+d_{v}}\right)^{2} \leq \frac{m^{2}}{4} \cdot \frac{\Delta^{2}+\delta^{2}}{\Delta \delta}
$$

This implies that

$$
\mathrm{GA}(G)^{2} \geq 4 M_{2}(G) \chi_{-2}(G)-\frac{m^{2}}{4} \cdot \frac{\Delta^{2}+\delta^{2}}{\Delta \delta} .
$$

The result follows.

Now, we obtain a lower bound for the geometricarithmetic index in terms of the harmonic index.

Theorem 18. Let $G$ be a graph without isolated edges. Then,

$$
\mathrm{GA}(G) \geq \sqrt{2} H(G)
$$

Proof. Since for each $u v \in E(G), d_{u} d_{v} \geq 2$, we obtain

$$
\mathrm{GA}(G)=\sum_{u v \in E(G)} \frac{2 \sqrt{d_{u} d_{v}}}{d_{u}+d_{v}} \geq \sum_{u v \in E(G)} \frac{2 \sqrt{2}}{d_{u}+d_{v}}=\sqrt{2} H(G),
$$

as desired.

The proof of next results can be found in [33].

Theorem 19 (see [33]). Let $G$ be a triangle-free graph of order $n$ and the minimum degree $\delta \geq k(k \leq(n / 2))$. Then,

$$
H(G) \geq \frac{2 k(n-k)}{n} \text {. }
$$

Theorem 20 (see [33]). Let $G$ be a triangle-free graph of order $n$ and size $m$. Then,

$$
H(G) \geq \frac{2 m}{n}
$$

Applying Theorems 18-20, it leads to the next results.

Corollary 14. Let $G$ be a triangle-free graph of order $n$ without isolated edges, and the minimum degree $\delta \geq k(k \leq(n / 2))$. Then,

$$
\begin{aligned}
& \mathrm{GA}(G) \geq \frac{2 \sqrt{2} k(n-k)}{n}, \\
& \mathrm{GA}(G) \geq \frac{2 \sqrt{2} m}{n} .
\end{aligned}
$$

We can see that Inequality (82) improves the next wellknown result for triangle-free graphs [13]. Let $G$ be a graph of order $n$ and size $m$ without isolated vertex. Then,

$$
\mathrm{GA}(G) \geq \frac{2 m}{n}
$$

The eccentricity $\varepsilon(v)$ of $v$ is defined as

$$
\varepsilon(v)=\max \{d(v, w): w \in V(G)\},
$$

where $d(v, w)$ is the length of a shortest path connecting $v$ and $w$. The radius $r$ and diameter $D$ are defined as the minimum and maximum values among $\varepsilon(v)$ over all vertices $v \in V(G)$, respectively.

$\mathrm{Xu}$ [34] showed that, for any nontrivial connected graph $G$ of order $n$, size $m$, and radius $r, H(G) \geq(m / n-r)$. Using this and Theorem 18, we obtain the next result.

Corollary 15. Let $G$ be a nontrivial connected graph of order $n$, size $m$, and radius $r$. Then,

$$
\mathrm{GA}(G) \geq \frac{\sqrt{2} m}{n-r}
$$

Theorem 21. Let $G$ be a nontrivial connected graph of size $m$ and radius $r$. Then,

$$
\mathrm{GA}(G) \geq \frac{R_{1 / 2}(G)}{n-r} .
$$

Proof. Note that, for each vertex $u \in V(G)$, we have $d_{u} \leq n-\varepsilon(u)$. Thus, for each edge $u v \in E(G)$,

$$
\begin{aligned}
\mathrm{GA}(G) & =\sum_{u v \in E(G)} \frac{2 \sqrt{d_{u} d_{v}}}{d_{u}+d_{v}} \geq \sum_{u v \in E(G)} \frac{2 \sqrt{d_{u} d_{v}}}{2 n-\varepsilon(u)-\varepsilon(v)} \\
& \geq \sum_{u v \in E(G)} \frac{2 \sqrt{d_{u} d_{v}}}{2 n-2 r}=\frac{R_{1 / 2}(G)}{n-r},
\end{aligned}
$$

as desired.

Theorem 22. Let $G$ be a nontrivial graph of order $n$, size $m$, and $p$ pendent edges without isolated vertex. Then,

$$
\mathrm{GA}(G) \geq \frac{p}{\sqrt{n-1}}+\frac{m-p}{n-1-(p / 2)} .
$$

Proof. Since $0<\left(1 / d_{u}\right)$ and $\left(1 / d_{v}\right) \leq 1$, therefore we deduce that

$$
\begin{aligned}
\mathrm{GA}(G) & =\sum_{u v \in E(G)} \frac{2 \sqrt{d_{u} d_{v}}}{d_{u}+d_{v}} \geq \sum_{u v \in E(G)} \frac{\left(\left(1 / d_{u}\right)+\left(1 / d_{v}\right)\right) \sqrt{d_{u} d_{v}}}{d_{u}+d_{v}} \\
& =\sum_{u v \in E(G)} \frac{1}{\sqrt{d_{u} d_{v}}} .
\end{aligned}
$$

For each pendent edge $e=u v$, we clearly have (1/ $\left.\sqrt{d_{u} d_{v}}\right) \geq(1 / \sqrt{n-1})$. If $e=u v$ is a nonpendent edge, then $d_{u}+d_{v} \leq 2(n-1)-p$, as any pendent vertex is adjacent to at most one of $u$ and $v$. So, $\sqrt{d_{u} d_{v}} \leq\left(d_{u}+d_{v} / 2\right) \leq n-1-(p / 2)$; hence, 


$$
\frac{1}{\sqrt{d_{u} d_{v}}} \geq \frac{1}{n-1-(p / 2)} .
$$

Thus,

$$
\mathrm{GA}(G) \geq \frac{p}{\sqrt{n-1}}+\frac{m-p}{n-1-(p / 2)}
$$

The desired result follows.

In [35], Kulli et al. defined the first and second generalized multiplicative Zagreb indices:

$$
\begin{aligned}
& M Z_{1}^{a}(G)=\prod_{u v \in E(G)}\left(d_{u}+d_{v}\right)^{a}, \\
& M Z_{2}^{a}(G)=\prod_{u v \in E(G)}\left(d_{u} d_{v}\right)^{a} .
\end{aligned}
$$

Here, we obtain a lower bound in terms of the first and second generalized multiplicative Zagreb indices.

Theorem 23. Let $G$ be a nontrivial graph of size $m$. Then,

$$
\mathrm{GA}(G) \geq 2 m \sqrt{\frac{M Z_{2}^{1 / 2}(G)}{M Z_{1}^{1}(G)} .}
$$

Proof. By Lemma 2, we obtain

$$
\begin{aligned}
\frac{\mathrm{GA}(G)}{2 m} & =\frac{1}{m} \sum_{u v \in E(G)} \frac{\sqrt{d_{u} d_{v}}}{d_{u}+d_{v}} \\
& \geq \sqrt{\prod_{u v \in E(G)} \frac{\sqrt{d_{u} d_{v}}}{d_{u}+d_{v}}} \\
& =\sqrt[m]{\frac{\prod_{u v \in E(G)} \sqrt{d_{u} d_{v}}}{\prod_{u v \in E(G)}\left(d_{u}+d_{v}\right)=\sqrt[m]{\frac{M Z_{2}^{1 / 2}(G)}{M Z_{1}^{1}(G)}}}}
\end{aligned}
$$

as desired.

Theorem 24. Let $G$ be a graph of size $m$ and minimum degree $\delta$. Then,

$$
\mathrm{GA}(G) \geq \frac{4 \delta^{2} m^{2}}{\operatorname{HM}(G)}
$$

$$
\begin{aligned}
\frac{\mathrm{GA}(G)}{2 m} & =\frac{1}{m} \sum_{u v \in E(G)} \frac{\sqrt{d_{u} d_{v}}}{d_{u}+d_{v}} \\
& \geq \frac{1}{m} \sum_{u v \in E(G)} \frac{\left(2 d_{u} d_{v} /\left(d_{u}+d_{v}\right)\right)}{d_{u}+d_{v}}=\frac{1}{m} \sum_{u v \in E(G)} \frac{2 d_{u} d_{v}}{\left(d_{u}+d_{v}\right)^{2}} \\
& \geq \frac{m}{\sum_{u v \in E(G)}\left(\left(d_{u}+d_{v}\right)^{2} / 2 d_{u} d_{v}\right)} \\
& \geq \frac{m}{\left(1 / 2 \delta^{2}\right) \sum_{u v \in E(G)}\left(d_{u}+d_{v}\right)^{2}} \\
& =\frac{2 \delta^{2} m}{\operatorname{HM}(G)}
\end{aligned}
$$

as desired.

In the sequel, we obtain a lower bound in terms of the first Zagreb index.

Theorem 25. Let $G$ be a graph of size $m$, maximum degree $\Delta$, and minimum degree $\delta$. Then,

$$
\mathrm{GA}(G) \geq \frac{\delta m}{\Delta}+2 m-\frac{M_{1}(G)}{\delta} .
$$

Proof. By Lemma 8, we have

$$
\begin{aligned}
\mathrm{GA}(G)+\frac{M_{1}(G)}{\delta} & \geq \sum_{u v \in E(G)}\left(\frac{2 \sqrt{d_{u} d_{v}}}{d_{u}+d_{v}}+\frac{d_{u}+d_{v}}{\sqrt{d_{u} d_{v}}}\right) \\
& \geq \sum_{u v \in E(G)}\left(\frac{2 \sqrt{d_{u} d_{v}}}{d_{u}+d_{v}}+2\right) \\
& =\sum_{u v \in E(G)} \frac{2 \sqrt{d_{u} d_{v}}}{d_{u}+d_{v}}+\sum_{u v \in E(G)} 2 \\
& \geq \frac{\delta m}{\Delta}+2 m,
\end{aligned}
$$

and this implies the desired bound.

Zhou [36] proved that, for any triangle-free graph of order $n$ and size $m, M_{1}(G) \leq m n$. Together with Theorem 25, we get the next result.

Corollary 16. Let $G$ be a triangle-free graph of order $n$, size $m$, maximum degree $\Delta$, and minimum degree $\delta$. Then,

$$
\mathrm{GA}(G) \geq m\left(\frac{\delta}{\Delta}+2-\frac{n}{\delta}\right) .
$$

Inequality (98) leads to the following results. 
Corollary 17. Let $G$ be a graph of size m, maximum degree $\Delta$, and minimum degree $\delta$. Then,

$$
\begin{aligned}
& \mathrm{GA}(G) \geq \delta H(G)+2 m-\frac{M_{1}(G)}{\delta}, \\
& \mathrm{GA}(G) \geq \frac{R_{1 / 2}(G)}{\Delta}+2 m-\frac{M_{1}(G)}{\delta} .
\end{aligned}
$$

Note that, for every two real numbers $x$ and $y$, $\left((x+y)^{2} / x y\right) \geq 4$. Applying this, we obtain a lower bound for the geometric-arithmetic index in terms of the hyperZagreb index.

Theorem 26. Let $G$ be a graph of size $m$, maximum degree $\Delta$, and minimum degree $\delta$. Then,

$$
\mathrm{GA}(G) \geq \frac{\delta m}{\Delta}+4 m-\frac{\operatorname{HM}(G)}{\delta^{2}} .
$$

Proof. From the above inequality, we have

$$
\begin{aligned}
\mathrm{GA}(G)+\frac{\operatorname{HM}(G)}{\delta^{2}} & \geq \sum_{u v \in E(G)}\left(\frac{2 \sqrt{d_{u} d_{v}}}{d_{u}+d_{v}}+\frac{\left(d_{u}+d_{v}\right)^{2}}{d_{u} d_{v}}\right) \\
& \geq \sum_{u v \in E(G)}\left(\frac{2 \sqrt{d_{u} d_{v}}}{d_{u}+d_{v}}+4\right) \\
& =\sum_{u v \in E(G)} \frac{2 \sqrt{d_{u} d_{v}}}{d_{u}+d_{v}}+\sum_{u v \in E(G)} 4 \\
& \geq \frac{\delta m}{\Delta}+4 m,
\end{aligned}
$$

and this implies the desired bound.

Here, we obtain a lower bound for the geometricarithmetic index in terms of the first Zagreb index.

Theorem 27. Let $G$ be a graph of size $m$ and minimum degree $\delta$. Then,

$$
\mathrm{GA}(G) \geq 2 m-\frac{M_{1}(G)}{2 \delta} \text {. }
$$

Proof. From the fact that $x+(1 / x) \geq 2$ for any $x>0$, we have

$$
\begin{aligned}
\mathrm{GA}(G)+\frac{M_{1}(G)}{2 \delta} & \geq \sum_{u v \in E(G)}\left(\frac{2 \sqrt{d_{u} d_{v}}}{d_{u}+d_{v}}+\frac{d_{u}+d_{v}}{2 \sqrt{d_{u} d_{v}}}\right) \\
& \geq \sum_{u v \in E(G)} 2=2 m,
\end{aligned}
$$

and this implies the desired bound.

Theorem 28 (see [37]). Let $G$ be a graph of size $m$ and diameter $D>1$. Then,

$$
M_{1}(G) \leq m^{2}-m(D-3)+(D-2) .
$$
result.

Now, by Theorems 27 and 28, we have the following

Corollary 18. Let $G$ be a graph of size $m$, minimum degree $\delta$, and diameter $D>1$. Then,

$$
\mathrm{GA}(G) \geq 2 m-\frac{m^{2}-m(D-3)+(D-2)}{2 \delta} .
$$

Theorem 29 (see [38]). Let $G$ be a graph of size $m$, with $t$ triangles and pendent vertex $p$. Then,

$$
M_{1}(G) \leq m(p+2)+3 t .
$$

Again, by Theorems 27 and 29, we have the following result.

Corollary 19. Let $G$ be a graph of size $m$, with $t$ triangles, leaf number $L$, and minimum degree $\delta$. Then,

$$
\mathrm{GA}(G) \geq 2 m-\frac{m(p+2)+3 t}{2 \delta} .
$$

Theorem 30 (see [39]). Let $G$ be a triangle- and quadranglefree graph with $n>1$ vertices. Then,

$$
M_{1}(G) \leq n(n-1) \text {. }
$$

Also, by Theorems 27 and 30, we have the following result.

Corollary 20. Let $G$ be a triangle- and quadrangle-free graph of order $n$, size $m$, and minimum degree $\delta$. Then,

$$
\mathrm{GA}(G) \geq 2 m-\frac{n(n-1)}{2 \delta} \text {. }
$$

\section{Data Availability}

The data used to support the findings of the study are provided within the article.

\section{Conflicts of Interest}

The authors declare that they have no conflicts of interests.

\section{References}

[1] H. Wiener, "Structural determination of paraffin boiling points," Journal of the American Chemical Society, vol. 69, no. 1, pp. 17-20, 1947.

[2] E. Estrada, L. Torres, L. Rodríguez, and I. Gutman, “An atombond connectivity index: modelling the enthalpy of formation of alkanes," Indian Journal of Chemistry, vol. 37A, pp. 849$855,1998$. 
[3] P. S. Ranjini, V. Lokesha, M. Bindusree, and M. P. Raju, "New bounds on Zagreb indices and the Zagreb co-indices," Boletim da Sociedade Paranaense de Matematica, vol. 31, pp. 51-55, 2013.

[4] H. Aram and N. Dehgardi, "Reformulated F-index of graph operations," Communications in Combinatorics Optimization, vol. 2, pp. 87-98, 2017.

[5] I. Gutman, M. K. Jamil, and N. Akhter, "Graphs with fixed number of pendent vertices and minimal first Zagreb index," Transactions on Combinatorics, vol. 4, pp. 43-48, 2015.

[6] I. Gutman and N. Trinajstić, "Graph theory and molecular orbitals. total $\pi$-electron energy of alternant hydrocarbons," Chemical Physics Letters, vol. 17, no. 4, pp. 535-538, 1972.

[7] A. Ali, M. Javaid, M. Matejić, I. Milovanović, and E. Milovanović, "Some new bounds on the general sum-connectivity index," Communications in Combinatorics and Optimization, vol. 5, pp. 97-109, 2020.

[8] B. Zhou and N. Trinajstić, "On general sum-connectivity index," Journal of Mathematical Chemistry, vol. 47, no. 1, pp. 210-218, 2010.

[9] G. H. Shirdel, H. Rezapour, and A. M. Sayadi, "The hyper Zagreb index of graph operations," Iranian Journal of Mathematical Chemistry, vol. 4, pp. 213-220, 2013.

[10] Z. Du, A. Jahanbai, and S. M. Sheikholeslami, "Relationships between randić index and other topological indices," Communications in Combinatorics Optimization, vol. 6, pp. 137154, 2021.

[11] S. Fajtlowicz, "On conjectures of grati-II," Congruent Number, vol. 60, pp. 187-197, 1987.

[12] B. Furtula and I. Gutman, "A forgotten topological index," Journal of Mathematical Chemistry, vol. 53, no. 4, pp. 1184$1190,2015$.

[13] D. Vukičević and B. Furtula, "Topological index based on the ratios of geometrical and arithmetical means of end-vertex degrees of edges," Journal of Mathematical Chemistry, vol. 46, pp. 1369-1376, 2009.

[14] K. C. Das, I. Gutman, and B. Furtula, "Survey on geometricarithmetic indices of graphs," MATCH Communication in Mathematical and in Computer Chemistry, vol. 65, pp. 595644, 2011.

[15] M. Mogharrab and G. H. Fath-Tabar, "Some bounds on $G A_{1}$ index of graph," MATCH Communication in Mathematical and in Computer Chemistry, vol. 65, pp. 33-38, 2011.

[16] Y. Yuan, B. Zhou, and N. Trinajstić, "On geometric-arithmetic index," Journal of Mathematical Chemistry, vol. 47, no. 2, pp. 833-841, 2010.

[17] I. J. Taneja, "Refinement of inequalities among means," Journal of Combinatorics Information System Science, vol. 31, pp. 343-364, 2006.

[18] D. S. Bernstein, Matrix Mathematics: Theory, Facts, and Formulas, Princeton University Press, New Jersy, NY, USA, 2009.

[19] J. Radon, "Theorie und anwendungen der absolut additiven mengenfunktionen," Sitzungsber. Acad. Wissen. Wien.vol. 122, pp. 1295-1438, 1913.

[20] A. Lupas, "A remark on the schweitzer and kantorovich inequalities," Univ. Beograd Publ. Elektrotehn. Fak. Ser. Mat. Fiz.vol. 381-409, pp. 13-15, 1972.

[21] N. Ozeki, "On the estimation of inequalities by maximum and minimum values," Journal College Arts Sciences, vol. 5, pp. 199-203, 1968.

[22] G. H. Hardy, J. E. Littlewood, and G. Pólya, Inequalities, Cambridge University Press, Cambridge, England, 1934.
[23] B. Bollobás and P. Erdös, "Graphs of extremal weights," Ars Combinatoria, vol. 50, pp. 225-233, 1998.

[24] B. Borovićanin and T. A. Lampert, "On the maximum and minimum Zagreb indices of trees with a given number of vertices of maximum degree," MATCH Communication in Mathematical and in Computer Chemistry, vol. 74, pp. 81-96, 2015.

[25] K. C. Das and I. Gutman, "Some properties of the second Zagreb index," MATCH Communication in Mathematical and in Computer Chemistry, vol. 52, pp. 103-112, 2004.

[26] S. Wang, W. Gao, M. K. Jamil, M. R. Farahani, and J. Liu, "Bounds of Zagreb indices and hyper Zagarb indices," Mathematical Reports, vol. 21, pp. 93-102, 2019.

[27] P. S. Ranjini, V. Lokesha, and A. Usha, "Relation between phenylene and hexagonal squeeze using harmonic index," International Journal of Applied Graph Theory, vol. 1, pp. 116-121, 2013.

[28] I. Gutman, M. Togan, A. Yurttas, A. S. Cevik, and I. N. Cangul, "Inverse problem for sigma index," MATCH Communication in Mathematical and in Computer Chemistry, vol. 79, pp. 491-508, 2018.

[29] V. R. Kulli, "F-indices of chemical networks," International Journal of Mathematical Archive, vol. 10, pp. 21-30, 2019.

[30] K. C. Das, K. Xu, and I. Gutman, "On Zagreb and harary indices," MATCH Communication in Mathematical and in Computer Chemistry, vol. 70, pp. 301-314, 2013.

[31] L. Zhong, "The harmonic index for graphs," Applied Mathematics Letters, vol. 25, no. 3, pp. 561-566, 2012.

[32] A. Emanuela, T. Došlić, and A. Ali, "Two upper bounds on the weighted harary indices," Discrete Mathematics Letters, vol. 1, pp. 21-25, 2019.

[33] J. Liu, "On the harmonic index of triangle-free graphs," Applied Mathematics, vol. 04, no. 08, pp. 1204-1206, 2013.

[34] X. Xu, "Relationships between harmonic index and other topological indices," Applied Mathematical Science, vol. 6, pp. 2013-2018, 2012.

[35] V. R. Kulli, B. Stone, S. Wang, and B. Wei, "Generalised multiplicative indices of polycyclic aromatic hydrocarbons and benzenoid systems," Zeitschrift für Naturforschung A, vol. 72, no. 6, pp. 573-576, 2017.

[36] B. Zhou, "Zagreb indices," MATCH Communication in Mathematical and in Computer Chemistry, vol. 52, pp. 113118, 2004.

[37] B. Liu and I. Gutman, "Upper bounds for Zagreb indices of connected graphs," MATCH Communication in Mathematical and in Computer Chemistry, vol. 55, pp. 439-446, 2006.

[38] M. J. Morgan and S. Mukwembi, "Bounds on the first Zagreb index, with applications," in Topics in Chemical Graph Theory, I. Gutman, Ed., pp. 215-228, University of Kragujevac, Kragujevac, Serbia, 2014.

[39] B. Zhou and D. Stevanović, "A note on Zagreb indices," MATCH Communication in Mathematical and in Computer Chemistry, vol. 56, pp. 571-578, 2006. 